\title{
Full-aperture backscatter measurements on the National Ignition Facility
}

\author{
D. H. Froula, ${ }^{a)}$ D. Bower, M. Chrisp, S. Grace, J. H. Kamperschroer, T. M. Kelleher, \\ R. K. Kirkwood, B. MacGowan, T. McCarville, N. Sewall, F. Y. Shimamoto, \\ S. J. Shiromizu, B. Young, and S. H. Glenzer \\ Lawrence Livermore National Laboratory, University of California, P.O. Box 808, Livermore, \\ California 94551
}

(Presented on 21 April 2004; published 13 October 2004)

\begin{abstract}
The National Ignition Facility's full-aperture backscatter station (FABS) is described. The FABS uses five independent diagnostics on each of the four laser beams in the initial National Ignition Facility quad to measure the energy, power, spectrum, and near-field amplitude modulations of the stimulated Brillouin and stimulated Raman backscattered light. In initial tests $\mathrm{CO}_{2}$ and $\mathrm{C}_{5} \mathrm{H}_{12}$ gas-filled targets were used to create various laser-plasma interaction conditions which have shown the capability of producing ignition size laser plasmas with reflectivites on the order of $10 \%$. Results are presented for tests in which $16 \mathrm{~kJ}$ on target produced between 0.3 and $2.5 \mathrm{~kJ}$ of backscattered light. () 2004 American Institute of Physics. [DOI: 10.1063/1.1789592]
\end{abstract}

\section{INTRODUCTION}

The National Ignition Facility (NIF) will employ several high intensity laser beams propagating through large-scalelength plasmas creating high energy density conditions suitable for inertial confinement fusion (ICF) implosions. Ignition designs are sensitive to the strict energy balance between the laser beams and require efficient energy coupling to the target. ${ }^{1}$ It is therefore important to temporally diagnose and control the energy backscattered from ignition targets. It is also important to understand backward propagating light in the NIF laser system as a potential source of system damage. ${ }^{2}$ The first full-aperture backscatter station (FABS) on NIF uses calorimeters, streaked spectrometers, fast photodiodes, near-field cameras, and time integrated spectrometers to independently measure the characteristics of both the stimulated Brillouin backscattered (SBS) and stimulated Raman backscattered (SRS) light for each of four incident laser beams.

\section{A. General characteristics of NIF backscattered light}

The FABS has been designed for a wide parameter space defined by the variety of expected targets and laser conditions that will be employed by the NIF. The laser has been designed to operate at an energy of $1.8 \mathrm{MJ}$ using 192 laser beams. The 192 beams are split into 48 quads (four beams) which can deliver up to $36 \mathrm{~kJ}$ of $3 \omega\left(\lambda_{0}=351 \mathrm{~nm}\right)$ laser light to the target chamber center. To date, a single quad and one FABS have been activated.

The FABS is designed to measure the light scattered from the target back through the final optics assembly (FOA). The primary mechanism for the backscattered light is stimulated scattering instabilities; a result of the resonant coupling of an intense laser pulse, a scattered light wave, and an ion-acoustic wave (SBS) or an electron plasma wave

\footnotetext{
${ }^{\text {a)} E l e c t r o n i c ~ m a i l: ~ f r o u l a 1 @ ~ @ l l n l . g o v ~}$
}

(SRS). ${ }^{3}$ In general, the resulting scattered light wave propagates directly back along the incident laser path. In the case of SBS, the wavelength of the light is primarily a function of the electron temperature and the macroscopic plasma motion and is only slightly redshifted from the lasers fundamental wavelength, $\left(\lambda_{\mathrm{SBS}}-\lambda_{0}\right)<4 \mathrm{~nm}$. On the other hand, the wavelength of the SRS light is dependent on the density at which the scattering process takes place and is typically a broad $60 \mathrm{~nm}$ peak centered between $\lambda_{\text {SRS }}=450-600 \mathrm{~nm}$. Typical ICF experiments measure SBS and SRS reflectivities as high as $30 \%$ and below $1 \%$ depending on the details of the target and the conditions of the laser beams.

\section{B. General FABS system design requirements}

The FABS has been designed to measure both SBS and SRS backscattered energies between $5 \mathrm{~J}$ and $2 \mathrm{~kJ}$ to within an uncertainty of 5\%. This requirement has led to the development of a novel calibration and measurement technique that uses time integrated spectrometers. The discussion of this system is presented in detail in Ref. 4. The SBS spectra is to be measured with a resolution of $0.5 \mathrm{~nm}$ within a wavelength range of $348-354 \mathrm{~nm}$ while the SRS spectra is to have a range of $400-700 \mathrm{~nm}$ with a $2.5 \mathrm{~nm}$ resolution. Both the spectral measurements are designed to have a temporal resolution of $50 \mathrm{ps}$ over a 10,30 , or $50 \mathrm{~ns}$ time window. Spatial amplitude modulations propagating through the FOA are to be resolved to $2 \mathrm{~mm}$.

\section{OPTICAL SETUP}

The FABS collects light that has been backscattered into the four $40 \mathrm{~cm} \times 40 \mathrm{~cm}$ square focusing lenses. The backscattered light is collimated by the lenses while passing through the FOA (Fig. 1). Each FOA consists of a debris shield, focusing lens, a doubler crystal, a tripler crystal, and a vacuum window. The FOA transmits about $85 \%$ of the backscattered light. About $60 \%$ of the backscattered light is then transmitted through the last turning mirror in the NIF 


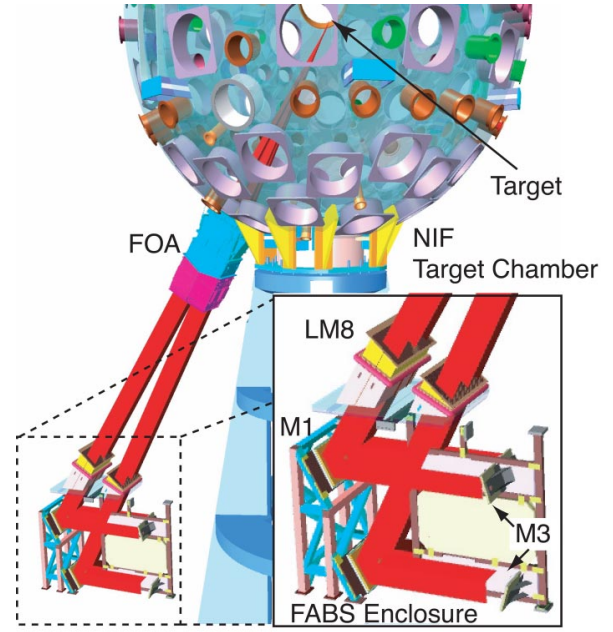

FIG. 1. (Color online) The light backscattered from target chamber center into the FOA is collected and transported through LM8 to the FABS enclosure.

laser chain (LM8) into the FABS enclosure. The full aperture beams are then reflected off uncoated glass (M1) at an angle of $32^{\circ}$ to the normal reducing the backscattered energy to about $2 \%$. The light is focused by a full aperture aluminum coated spherical mirror (M3) through a beam splitter (BS1) that discriminates between SBS and SRS light. BS1 is coated to reflect nearly $100 \%$ of the light below $400 \mathrm{~nm}$ (SBS) while transmitting light of higher wavelength (SRS). A fullaperture absorbing glass filter can be inserted between M1 and M3 to reduce the fluence on M3 and BS1 during high energy backscatter shots.

Figure 2 shows the optical setup for the SBS light reflected off BS1. The SRS light transmitted through BS1 follows a similar path to the one shown here. Light is imaged onto the SBS calorimeter after reflecting off an uncoated glass beam splitter (BS4). A large area filter wheel selects the down stream energy density using up to five absorbing glass neutral density filters. In theory, the filtering on all individual

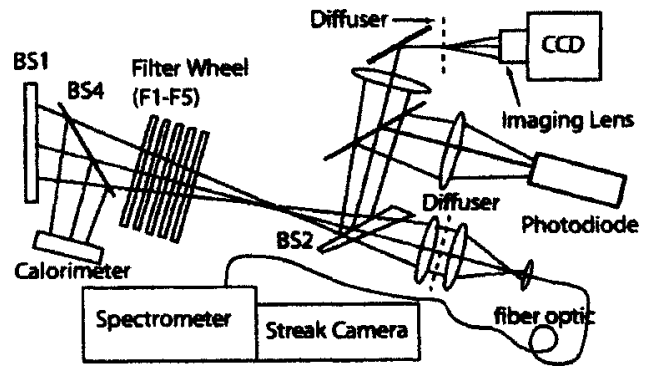

FIG. 2. The optical layout for one side (SBS or SRS) of a single beam is shown.

diagnostics can be set so that each sensitivity is equivalent, therefore, the filter wheel is the only filtering that is changed between shots.

Three reflections from the FOA propagate into the FABS: the back surface of the doubler crystal ( $2 \omega$ light), front surface of the tripler crystal ( $2 \omega$ light), and the back surface of the tripler crystal $(3 \omega$ light and unconverted $2 \omega$ light). The $2 \omega$ light reflected off of the doubler crystal counter propagates colinear with the incident laser and is optically filtered out by BS1 in the case of the SBS diagnostics, and temporally gated out in the case of the SRS diagnostics. The reflections from the tripler crystal are spatially filtered at the focus of M3; these reflections propagate slightly off axis due to a slight angle in the tripler crystal.

The SBS near-field light is imaged onto a diffuser using a combination of the focusing mirror (M3) and a planocylindrical lens. A standard camera lens images the diffuser onto a 768 pixel $\times 512$ pixel charge coupled device camera.

The SBS and SRS spectra are recorded using streak cameras coupled to spectrometers. The light is coupled to the spectrometers using an $8 \mathrm{~m} 100 \mu \mathrm{m}$ fiber. A diffuser plate is used to remove spatial intensity modulations allowing reliable coupling into the fiber. A simple optical system relays the light transmitted through the diffuser plate on to the end of the fiber optic.

TABLE I. Details for each diagnostic are given. A summary of the measured system performance is presented: time resolution $(\Delta t)$, wavelength range $(\lambda)$, wavelength resolution $(\Delta \lambda)$, spatial resolution $(\Delta x)$. The energy range for each system is determined by the diagnostic, total optical transmission, neutral density filters, and the filter wheel.

\begin{tabular}{|c|c|c|c|c|c|c|c|}
\hline Diagnostic details & Manufacturer & Model No. & Energy & $\Delta t$ & $\Delta x$ & $\lambda$ & $\Delta \lambda$ \\
\hline Calorimetry system performance & & & $1 \mathrm{~J}-2.5 \mathrm{~kJ}$ & integrated & integrated & pass $<400 \mathrm{~nm}$ & \\
\hline SBS/SRS Cal's & Coherent & J50LP-2A & $3 \mu J-7 \mathrm{~mJ}$ & integrated & integrated & & \\
\hline SBS photodiode system performance & & & $10 \mathrm{~mJ}-2.5 \mathrm{~kJ}$ & 200 ps & integrated & pass $<400 \mathrm{~nm}$ & \\
\hline SBS diodes & Hamamatsu & R1328U-51 & $2 \mathrm{~nJ}-0.1 \mu J$ & $60 \mathrm{ps}$ & integrated & $300-1100 \mathrm{~nm}$ & \\
\hline Digital oscilliscope & Tektronics & TDS-694 & & $3 \mathrm{GHz}$ & & & \\
\hline SBS near-field system performance & & & $1 \mathrm{~J}-2.5 \mathrm{~kJ}$ & integrated & $2 \mathrm{~mm}^{\mathrm{b}}$ & pass $<400 \mathrm{~nm}$ & \\
\hline SBS CCD & Finger Lakes & KAF-0401E & & integrated & $9 \mu m$ pxls & & \\
\hline SBS streak-spectrometry system performance & & & $10 \mathrm{~J}-2.5 \mathrm{~kJ}$ & $100 \mathrm{ps}^{\mathrm{b}}$ & integrated & $6.5 \mathrm{~nm}$ & $0.13 \mathrm{~nm}$ \\
\hline Streak camera & Hamamatsu & C7700-S20 & & $1 \%^{\mathrm{a}}$ & integrated & $300-850 \mathrm{~nm}$ & $0.0065 \mathrm{~nm} / \mathrm{pxl}$ \\
\hline $0.5-\mathrm{M}$ spectrometer & Acton & SP-500i & & $95 \mathrm{ps}$ & integrated & $250-500 \mathrm{~nm}$ & $1.7 \mathrm{~nm} / \mathrm{mm}$ \\
\hline SRS streak-spectrometry system performance & & & $10 \mathrm{~J}-2.5 \mathrm{~kJ}$ & $50 \mathrm{ps}^{\mathrm{b}}$ & integrated & $375 \mathrm{~nm}$ & $4.4 \mathrm{~nm}$ \\
\hline Streak camera & Hamamatsu & C7700-S1 & & $1 \%^{\mathrm{a}}$ & integrated & $400-1200 \mathrm{~nm}$ & 0.37 \\
\hline $0.15 \mathrm{M}$ spectrometer & Acton & SP-150 & & $10 \mathrm{ps}$ & integrated & $400-700 \mathrm{~nm}$ & $15 \mathrm{~nm} / \mathrm{mm}$ \\
\hline
\end{tabular}

${ }^{\mathrm{a}}$ The resolution is a multiple of the total time windows: $5,10,30$, and $50 \mathrm{~ns}$.

${ }^{\mathrm{b}} \mathrm{Has}$ not been experimentally verified. 

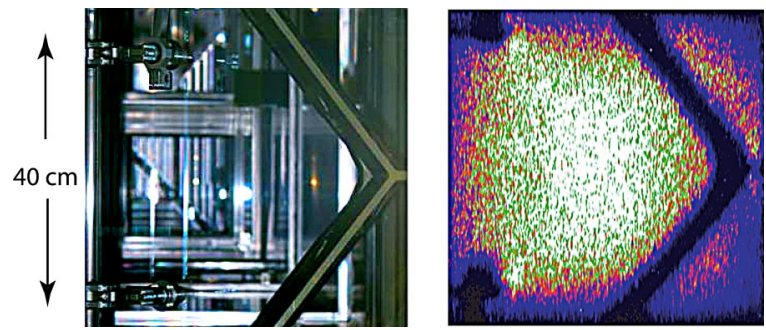

FIG. 3. (Color online) (Left) SBS $(10 \%)$ and (right) SRS $(<1 \%)$ spectral results obtained using the streaked diagnostic.

\section{DIAGNOSTIC DETAILS}

Table I summarizes the details for each diagnostic and the overall systems performance. Each system has been designed to meet the specific design requirements discussed in Sec. I B. The calorimeters are used to measure the total SBS and SRS energy. The calorimeters are prior to the beam blocks and do not discriminate between light backscattered from the target and the $\sim 1 \%$ reflections from the FOA. It is therefore necessary to subtract a precharacterized background. The calorimeters measure the backscattered energy to $20 \%$; this uncertainty is a combination of the optical transmission, subtraction of the FOA reflections, and electrical signal noise. Due to the damage threshold of the calorimeters $\left(250 \mathrm{~mJ} / \mathrm{cm}^{2}\right)$, it is necessary to use neutral density filters for backscattered energies above $30 \mathrm{~J}$. The photodiodes are used to time resolve the backscattered light and confirm that all reflections into the near-field camera are blocked. The streakspectrometer systems independently measure the SBS and SRS spectral behavior as a function of time.

\section{INITIAL BACKSCATTER RESULTS}

The FABS was activated in an incremental process; the laser energy was increased slowly to $3.8 \mathrm{~kJ}$. Low energy shots employed gold foil targets which produced low levels of backscatter and that allowed instruments to be timed and reflections into the FABS enclosure discriminated and properly blocked. $\mathrm{CO}_{2}$ gas-filled targets that produce high levels of SBS and $\mathrm{C}_{5} \mathrm{H}_{12}$ gas-filled targets that produce high levels of SRS were used to activate respective diagnostics. Gasfilled targets between 2 and $7 \mathrm{~mm}$ length allowed the backscattered energy to be scaled from $300 \mathrm{~J}$ to $2 \mathrm{~kJ}$. The targets were heated on one side by overlapping the $500 \mu \mathrm{m}$ focal spots of the four beams with a maximum total intensity of $2 \times 10^{15} \mathrm{~W} \mathrm{~cm}^{-2}$. The targets were filled with 1 atm of gas producing homogeneously heated plasmas with densities of $\mathrm{n}_{e}=6 \times 10^{20} \mathrm{~cm}^{-3}$ and temperatures of $\mathrm{T}_{e}=2 \mathrm{keV}$.
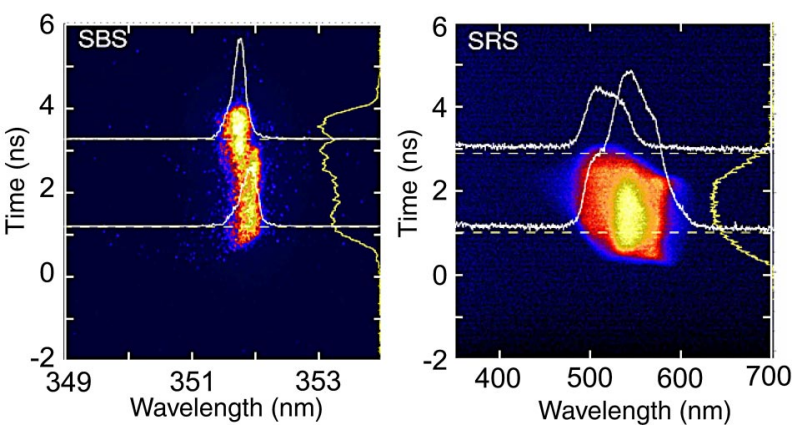

FIG. 4. (Color online) (Left) A picture of the FOA for a single beam (B318) as viewed by the FABS shows the LM8 mirror supports that block about $15 \%$ of the backscattered light. (Right) An SBS near-field image of the FOA shows the backscattered light peaked in the center of the lens with a modulation less than $3: 1$.

Concurrently, the FABS measured the backscatter energy, power, spectrum, and near-field images. The following presents the data for a shot where the laser delivered $3.8 \mathrm{~kJ}$ per beam on a $7 \mathrm{~mm} \mathrm{CO}$ gas-filled target. Figure 3 shows results of the streak camera data for the SBS and SRS systems. The calorimeters measured a total backscatter energy of $1.3 \mathrm{~kJ}$.

Figure 4 shows the SBS near-field image where the mirror structure that holds the last turning mirror (LM8) in the NIF laser chain is clearly evident. During the activation of this diagnostic the SBS amplitude modulations remained less than $3: 1$. In order to ramp up the backscattered energy, the amplitude modulations in the SBS light were closely monitored to prevent peak fluences above $\sim 2.5 \mathrm{~J} \mathrm{~cm}^{-2}$. During these shots the SBS photodiodes showed no significant light reflected from the crystals indicating that the light measured by the near-field cameras is predominantly from the target chamber center.

\section{ACKNOWLEDGMENT}

This work was performed under the auspices of the U.S. Department of Energy by the Lawrence Livermore National Laboratory under Contract No. W-7405-ENG-48.

${ }^{1}$ J. D. Lindl, P. A. Amendt, R. L. Berger, S. G. Glendinning, S. H. Glenzer, S. W. Haan, R. L. Kauffman, O. L. Landen, and L. J. Suter, Phys. Plasmas 11, 339 (2004).

${ }^{2}$ P. E. Young, Phys. Rev. Lett. 73, 1939 (1994).

${ }^{3}$ W. L. Kruer, The Physics of Laser Plasma Interactions (Addison-Wesley, Reading, MA, 1988).

${ }^{4}$ R. K. Kirkwood, T. McCarville, B. Young, M. Schneider, D. H. Froula, D. Bower, F. Holdener, M. Chrisp, B. MacGowan, and S. H. Glenzer, Rev. Sci. Instrum. (Submitted).

${ }^{5} \mathrm{~S}$. H. Glenzer et al., Proceedings of the 3rd International Conference on Inertial Fusion Sciences and Applications, Monterey, CA, 2004. 
Review of Scientific Instruments is copyrighted by the American Institute of Physics (AIP). Redistribution of journal material is subject to the AIP online journal license and/or AIP copyright. For more information, see http://ojps.aip.org/rsio/rsicr.jsp Copyright of Review of Scientific Instruments is the property of American Institute of Physics and its content may not be copied or emailed to multiple sites or posted to a listserv without the copyright holder's express written permission. However, users may print, download, or email articles for individual use. 